\title{
ANÁLISIS DE LA APTITUD RECREATIVA ORIENTADA A LA GESTIÓN AMBIENTAL DE LA PLAYA EL COCHÓRIT, SONORA, MÉXICO
}

\author{
Gisela GARCÍA-MORALES ${ }^{1}$, José Alfredo ARREOLA-LIZÁRRAGA ${ }^{1 *}$, \\ Renato Arturo MENDOZA-SALGADO ${ }^{2}$, Pedro ROSALES-GRANO ${ }^{3}$, Jaqueline GARCÍA-HERNÁNDEZ ${ }^{4}$, \\ Gustavo PADILLA-ARREDONDO ${ }^{1}$ y Alfredo ORTEGA-RUBIO ${ }^{2}$
}

${ }^{1}$ Centro de Investigaciones Biológicas del Noroeste, S.C. Carretera a Las Tinajas, kilómetro 2.3, predio El Tular, Guaymas, Sonora, México, C. P. 85454

${ }^{2}$ Centro de Investigaciones Biológicas del Noroeste, S.C. Instituto Politécnico Nacional 195, Playa Palo de Santa Rita Sur, La Paz, Baja California Sur, México, C. P. 23096

${ }^{3}$ Instituto Tecnológico de Guaymas. Carretera al Varadero Nacional, kilómetro 4, Colonia Las Playitas, Guaymas, Sonora, México, C. P. 85480

${ }^{4}$ Centro de Investigación en Alimentación y Desarrollo A.C. Carretera al Varadero Nacional, kilómetro 6.6, Colonia Las Playitas, Guaymas, Sonora, México, C. P. 85480

*Autor para correspondencia: aarreola04@cibnor.mx

(Recibido julio 2015; aceptado agosto 2016)

Palabras clave: playas recreativas, manejo de playas, indicadores ambientales, percepción pública

\section{RESUMEN}

La evaluación de la aptitud recreativa de playas es un asunto clave para diseñar estrategias de manejo. El objetivo de este estudio fue evaluar la aptitud recreativa y proponer una estrategia de manejo en la playa El Cochórit, municipio de Empalme, Sonora. La aptitud recreativa de la playa se evaluó con base en la percepción de los usuarios y en indicadores biofísicos. El valor estimado de la aptitud recreativa de la playa, según la percepción de los usuarios fue de 0.71 (medio) y con base en los indicadores biofísicos fue de 0.47 (medio). La aptitud recreativa de la playa requiere ser mejorada con atención prioritaria en: (1) inversión en infraestructura y servicios, (2) mantenimiento de limpieza, (3) implementación de programas de educación ambiental y (4) monitoreo anual de la calidad sanitaria del agua y de la arena. La estrategia de gestión ambiental debe considerar incluir a la playa El Cochórit en el Programa Playas Limpias de la Comisión Nacional del Agua con el propósito de orientar esfuerzos hacia su certificación como "playa limpia".

Key words: recreational beaches, management of beaches, environmental indicators, public perception

\begin{abstract}
The assessment of recreational beaches fitness is a key issue to design management strategies. The aim of this work was to evaluate the recreational fitness and propose a management strategy for the Beach El Cochórit, municipality of Empalme, Sonora. The recreational beach fitness was evaluated based on the perception of users, as well as biophysical indicators. The estimated value of recreational suitability of the beach perceived by users was 0.71 (average) and based on the biophysical indicators was
\end{abstract}


0.47 (average). Fitness recreational beach needs to be improved focusing on: (1) investing in infrastructure and services, (2) maintaining cleanliness, (3) implementing environmental education programs and (4) monitoring annually the sanitary quality of water and sand. The environmental management strategy should consider including the beach El Cochorit in the Clean Beaches Program of the National Water Commission in order to address efforts towards the certification of "clean beach".

\section{INTRODUCCIÓN}

Las playas recreativas requieren de evaluaciones y de un manejo orientados a mantener los servicios ecosistémicos que brindan a la sociedad. En ausencia de manejo, las playas recreativas han mostrado efectos adversos al ambiente y a la salud pública, así como a los ingresos económicos y a los empleos por turismo (Williams y Micallef 2009, Lucrecy et al. 2016). Contribuciones acerca del manejo de playas incluyen la seguridad física para los usuarios (Nelson et al. 2000, Micallef et al. 2004), la calidad sanitaria del agua y de la arena para determinar riesgos a la salud pública (Pereira et al. 2003, Silva-Iñiguez y Fisher 2003, Duvat 2012, Pinto et al. 2012, Rippy et al. 2013, Quilliam et al. 2015), valores estéticos, considerando aspectos de limpieza e higiene del espacio recreativo de las playas (Cervantes et al. 2008, Pranzini y Vitale 2011, Anfuso et al. 2014) prestación de servicios turísticos (Botero et al. 2013) y gestión con base en el ecosistema (Harris et al. 2015, Sardá et al. 2015), que en conjunto muestran la necesidad de generar conocimiento científico orientado a la atención de problemas que afectan el uso recreativo y mantenimiento saludable de las playas.

En las evaluaciones de playas se han aportado métodos basados en índices sobre la idoneidad de la playa para uso recreativo (Hernández 2003, Espejel et al. 2007, Popoca y Espejel 2009), así como la opinión de los usuarios en evaluaciones integrales (Cervantes y Espejel 2008, Roca et al. 2008, Lubinsky et al. 2009, Vaz et al. 2009, Navarro et al. 2012) para apoyar la gestión de su uso recreativo.

En México, la norma NMX-AA-120-SCFI-2016, establece los requisitos y especificaciones de sustentabilidad de calidad de playas (SEMARNAT 2016) y considera 52 indicadores en siete grupos de atributos: 1) calidad bacteriológica del agua, 2) residuos sólidos, 3) infraestructura costera, 4) biodiversidad, 5) seguridad y servicios, 6) educación ambiental y 7) contaminación por ruido. Actualmente, existen 33 playas certificadas, de las cuales 19 cuentan con la certificación internacional "Bandera Azul" (CONAGUA 2015).
En el Estado de Sonora existen aproximadamente 30 playas recreativas y ninguna está certificada, por lo que resulta importante realizar evaluaciones orientadas a su certificación y manejo. El objetivo de este estudio fue evaluar la aptitud recreativa para sustentar la gestión ambiental de la playa El Cochórit, municipio de Empalme, Sonora, México.

\section{MATERIALES Y MÉTODOS}

\section{Área de estudio}

La playa El Cochórit $(\sim 3.5 \mathrm{~km})$, está ubicada en el municipio de Empalme, Sonora, México (Fig. 1), con valores promedio anual de temperatura de $25^{\circ} \mathrm{C}$, precipitación de $\sim 300 \mathrm{~mm}$ y evaporación de $\sim 2600 \mathrm{~mm}$ (García 2004), las lluvias ocurren de julio a octubre con mayor magnitud en agosto (Douglas et al. 1993).

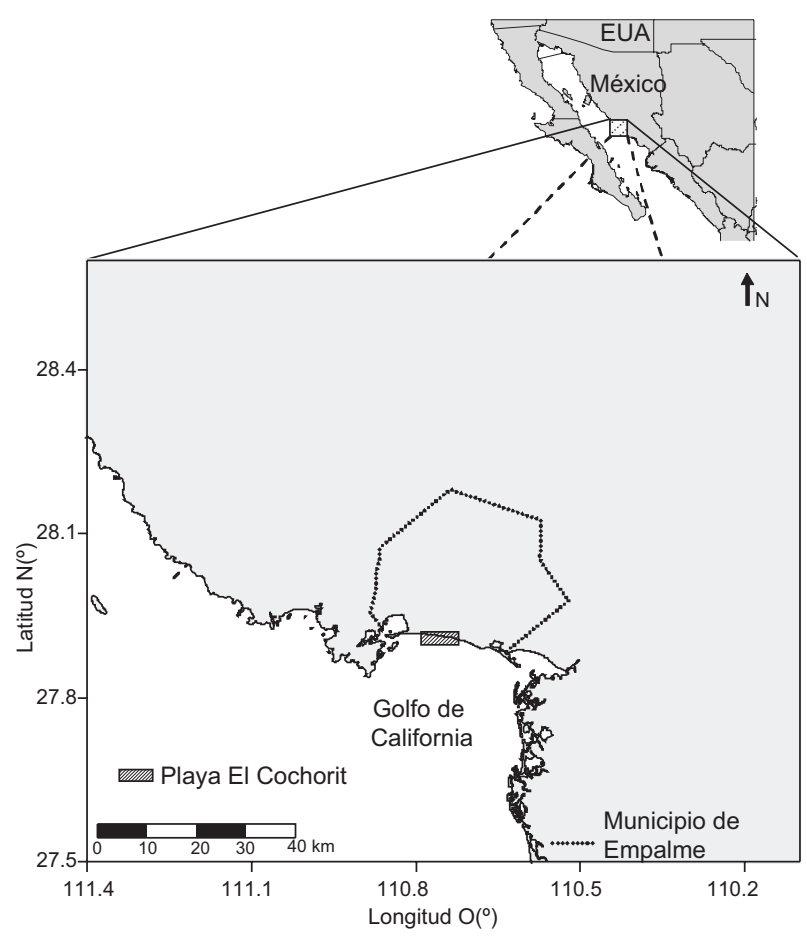

Fig. 1. Localización de la playa El Cochórit, municipio de Empalme, Sonora 
La playa El Cochórit está ubicada en la porción sur de un valle tectónico aluvial (Roldán-Quintana et al. 2004) comprendido en la subcuenca RH9-Cd río Mátape con superficie de drenaje de $720 \mathrm{~km}^{2}$ (INEGI 1993). La arena de la playa se deriva de los sedimentos que rellenan el valle, los cuales son predominantemente líticos de origen volcánico. La playa es somera, de baja pendiente y está limitada por dunas activas de baja altura y planicies de inundación. La energía de oleaje es baja y desarrolla bermas con elevación menor a un metro, por ello no hay gravas ni guijarros (Mendoza-Cantú 1997). Los principales usuarios de la playa El Cochórit son los habitantes de los municipios de Empalme y Guaymas, Sonora.

\section{Evaluación de la aptitud recreativa de la playa con base en la percepción de los usuarios}

Se diseñaron encuestas siguiendo los criterios de Cervantes et al. (2008) y se aplicaron a los usuarios de la playa para conocer tres aspectos: (1) perfil del usuario: edad, escolaridad, sexo, estado civil, ocupación y origen, (2) hábitos de recreación: temporada preferida para visitar la playa, motivos para visitar la playa y quiénes los acompañan, (3) opinión de las condiciones biofísicas y de infraestructura en la playa: características de la playa en los ambientes marino y terrestre, así como servicios disponibles. El número de encuestas se estimó mediante el método de curvas de isovarianza (Cochran y Cox 1965). Se obtuvo que 77 encuestas correspondían a una muestra representativa de la percepción de los usuarios sobre la playa. Con base en lo anterior, se aplicaron 80 encuestas, los datos obtenidos se capturaron en una hoja de cálculo EXCEL ${ }^{\circledR} 2010$.

La evaluación se realizó integrando las respuestas de la percepción de los usuarios en tres componentes: (1) infraestructura y servicios, (2) ambiental y (3) limpieza. A cada respuesta se le asignó un valor en una escala del 1 al 5 a partir de las características de la denominada "playa ideal", como lo sugieren Popoca y Espejel (2009). La calificación de la aptitud recreativa de la playa se obtuvo con el método de valoración de Micallef et al. (2004), en el cual se obtiene el porcentaje de frecuencia de cada una de las respuestas y la calificación se otorga a la que tenga la frecuencia más alta. Posteriormente, el valor de la calificación se divide entre cinco (el valor más alto en esta escala de evaluación), estos resultados se suman y el valor obtenido se divide entre el número de indicadores o preguntas evaluados de cada componente. De esta forma, los resultados se ubican en el intervalo que se muestra en el cuadro I.
CUADRO I. INTERVALOS DEAPTITUD RECREATIVA DE LA PLAYA

\begin{tabular}{ll}
\hline Aptitud recreativa & Intervalos \\
\hline Baja & $0.20-0.46$ \\
Media & $0.47-0.73$ \\
Alta & $0.74-1.00$ \\
\hline
\end{tabular}

\section{Evaluación de la aptitud recreativa de la playa por indicadores biofísicos}

Esta evaluación se realizó con base en la ficha descriptiva propuesta por Popoca y Espejel (2009) de la llamada "playa ideal". La ficha consta de 40 indicadores agrupados en los componentes: (1) infraestructura y servicios (14 indicadores), ambiental (18 indicadores) y limpieza (8 indicadores). La descripción se realizó a partir de observaciones directas y mediciones durante ocho fines de semana en el periodo vacacional de verano 2014. Específicamente, en el componente ambiental se realizaron perfiles de playa y granulometría de sedimentos. Se llevaron a cabo tres perfiles de playa separados entre sí cada $100 \mathrm{~m}$ que comprendieron desde las dunas hasta la zona intermareal. Para ello se establecieron controles horizontales con base en poligonales abiertas, así como controles verticales tomados del nivel del mar y predicciones de marea siguiendo los criterios de Casanova-Matera (2002). Las muestras de sedimentos fueron recolectadas en la parte superior, media e inferior de cada perfil de playa. Los sedimentos fueron analizados con un equipo láser de partículas modelo Horiba. El análisis granulométrico del sedimento se realizó mediante un histograma de frecuencia donde los intervalos de diámetro de partícula están expresados en unidades Phi $(-\log 2$ del diámetro de partícula expresado en $\mathrm{mm}$ ) como se describe en Folk y Ward (1956) y Royse (1970).

La evaluación consistió en asignar una calificación del 1 (no ideal) al 5 (ideal) a cada indicador. Los valores asignados se sumaron y el resultado obtenido fue dividido entre el número de indicadores evaluados en cada componente. La aptitud se asignó con base en los criterios mostrados en el cuadro I.

\section{RESULTADOS}

\section{Evaluación mediante la percepción de usuarios}

La mayoría de los usuarios de la playa son de origen nacional, con edades de 20-39 años, en los que predominan los de niveles escolares de secundaria y licenciatura (Fig. 2a). Los usuarios van en 


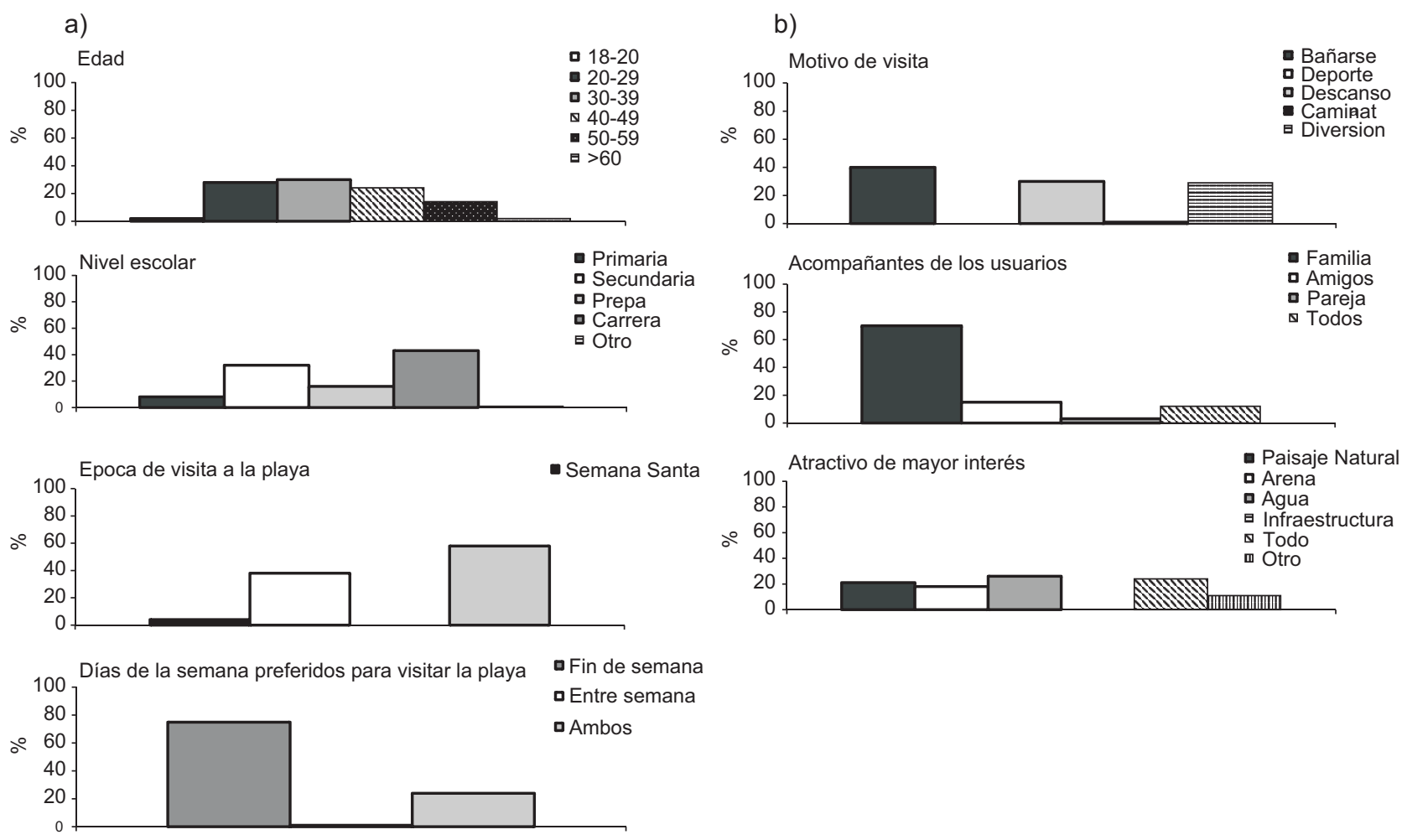

Fig. 2. Perfil de los usuarios (a) y hábitos de recreación (b) en la playa El Cochórit, Sonora

familia, visitan la playa en cualquier época del año, preferentemente el fin de semana, los principales motivos son diversión, baño y descanso en el que el atractivo principal es el agua (Fig. 2b).

En la playa la basura generada es transportada, en su mayor parte, por los usuarios, quienes la depositan en los contenedores de sus casas. Los accesos a la playa son fáciles pero escasos, y los servicios públicos e infraestructura en la playa (baños, estacionamiento y regaderas) son insuficientes. (Fig. 3a). La limpieza de la arena fue regular y mala. En ocasiones el agua o la arena tienen olores desagradables, eventualmente se observan animales (principalmente perros) merodeando en la playa. (Fig. 3b). A los usuarios les gusta la arena. La playa es considerada físicamente segura, con temperatura del agua agradable, no muy profunda, tiene oleaje regular y se advierte la presencia de insectos (Fig. 3c).

\section{Evaluación mediante indicadores biofísicos \\ Componente infraestructura y servicios}

El acceso a la playa se encuentra en buen estado y el estacionamiento no está zonificado, se carece de servicios públicos como sanitarios, regaderas, contenedores de basura, servicio de vigilancia y salvavidas. No hay áreas establecidas para realizar deportes playeros (volibol, futbol, etc.). Existe comercio minorista (vendedores ambulantes), se carece de malecones, muelles o alguna otra infraestructura vial, excepto la carretera a la playa. No hay información pública ni señalización permanente. Este componente obtuvo una calificación intermedia (0.51).

\section{Componente ambiental}

Es una playa estable, recta, con perfil morfodinámico intermedio-disipativo. El ancho de la playa promedio es de $15 \mathrm{~m}$ al nivel medio del mar, con longitud de $\sim 3.5 \mathrm{~km}$. Los perfiles de playa mostraron un relieve de pendiente suave y moderada de $4.3 \pm 0.7$ m (Fig. 4a), con profundidad de $2 \mathrm{~m}$ a los $15 \mathrm{~m}$ de distancia, la profundidad inmediata tras la rompiente de la ola es $<1 \mathrm{~m}$. La composición granulométrica de los sedimentos mostró que la playa tiene arenas finas cuyo diámetro predominante es $\varnothing=2.1$ ( 0.13 a 0.18 $\mathrm{mm}$ de diámetro), moderadamente seleccionados ya que el $72 \%$ está distribuido en seis tamaños de clase de 20 intervalos que componen la serie granulométrica (Fig. 4b). Debido a que la fuente de los sedimentos es principalmente de tierra, su coloración es café. La temperatura del agua tiene un intervalo anual de $18-30^{\circ} \mathrm{C}$. La amplitud de marea es de $0.85 \mathrm{~m}$ y la mayor parte del año el oleaje es $<1 \mathrm{~m}$ (Rosales-Grano 


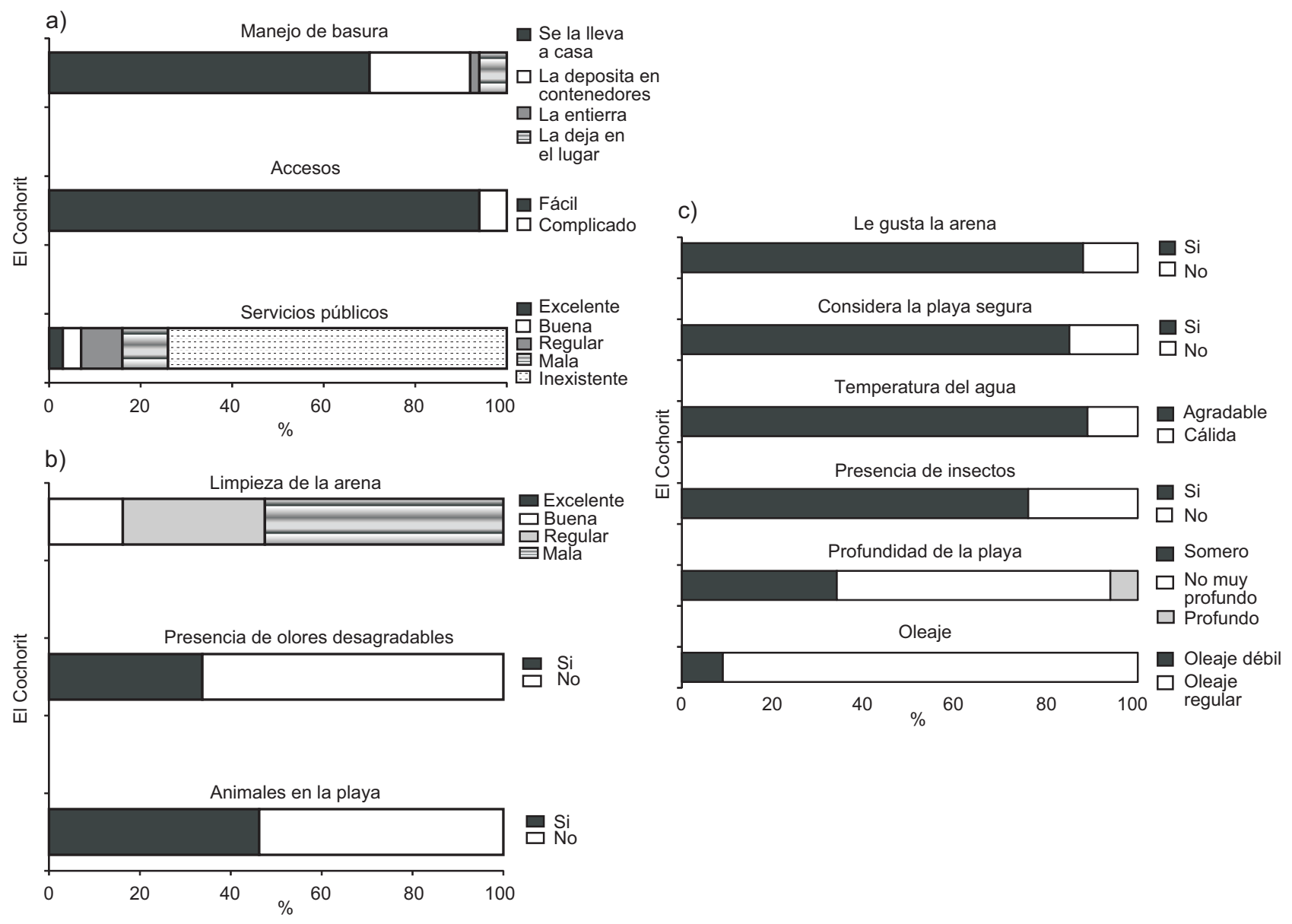

Fig. 3. Percepción de usuarios en: a) infraestructura y servicios, b) limpieza, c) ambiente de la playa El Cochórit, Sonora

2008), todo lo anterior califica a la playa con costa micromareal y oleaje bajo.

El ecosistema costero inmediato está en buen estado, con un campo visual amplio. La mayoría de los indicadores evaluados en este componente no responden a la calificación que coincide con la "playa ideal", aunque se puede categorizar como apta para realizar la actividad turística. La calificación del componente ambiental fue media (0.53).

\section{Componente de limpieza}

Frecuentemente, durante las visitas de observación a la playa se percibieron olores desagradables en la arena por basura excesiva, heces de animales domésticos y residuos sólidos. No se observaron derrames de aceites o hidrocarburos, ni tampoco descargas al mar de aguas residuales. La calidad sanitaria del agua durante y después del periodo vacacional de semana santa tuvo concentraciones de bacterias enterococos $>200$ del número más probable (NMP)/100 mL
(León-López et al. 2014), es decir con riesgos a la salud pública. La calificación del componente de limpieza fue bajo (0.37).

Con base en la integración de las observaciones de la percepción de los usuarios y la determinación de los aspectos biofísicos, la aptitud recreativa de la playa El Cochórit fue media (Cuadro II).

\section{DISCUSIÓN}

La evaluación de la aptitud recreativa de la playa El Cochórit integrada con el análisis de la percepción de los usuarios y los indicadores biofísicos fueron consistente para orientar decisiones de gestión ambiental.

\section{Percepción de usuarios}

La playa El Cochórit es visitada por usuarios para diversión, principalmente baño y descanso. Sin 
a) Perfiles de playa
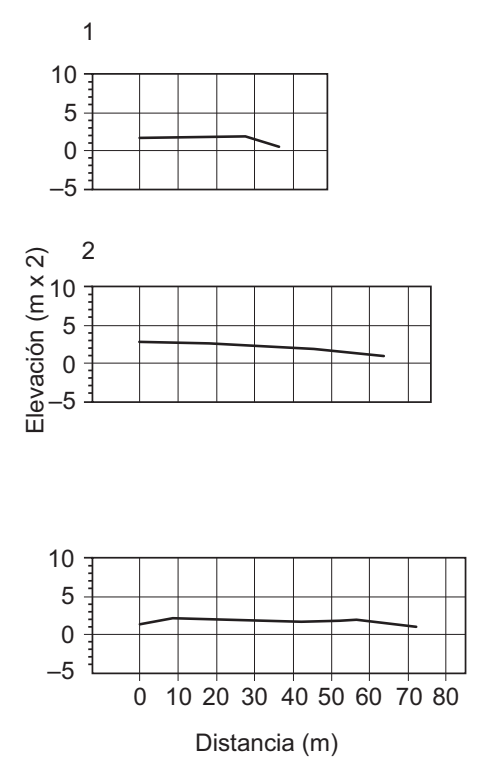

b) Distribución granulométrica de sedimentos

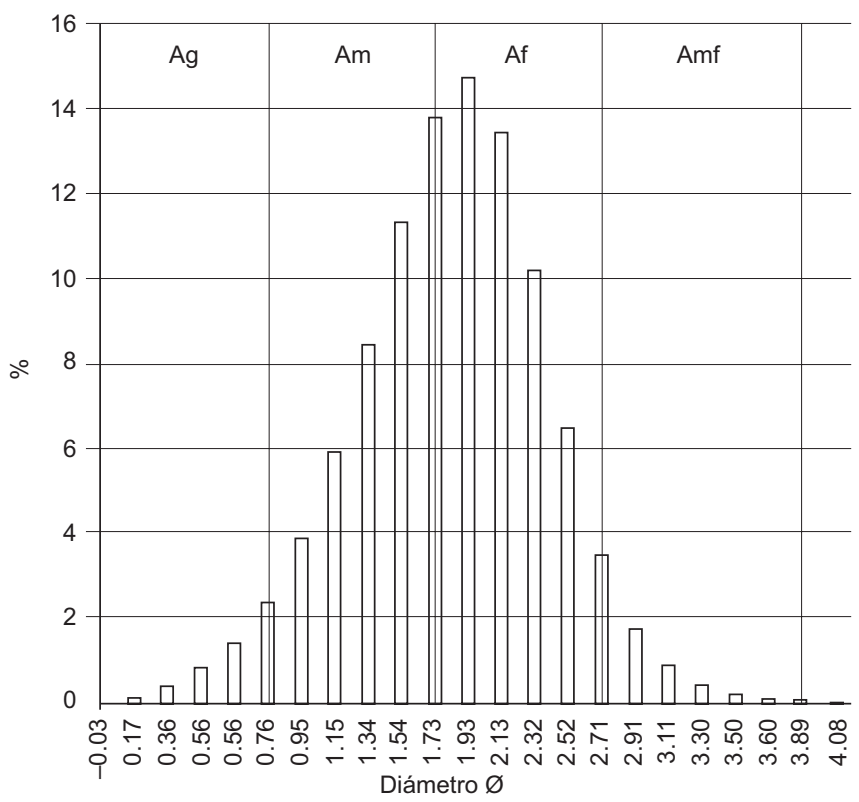

Fig.4. (a) perfiles y (b) composición granulométrica de la playa El Cochórit. Ag = Arena gruesa, Am = Arena media, Af = Arena fina y Amf = Arena muy fina. Las unidades $\varnothing$ son $-\log _{2}$ del diámetro de partícula en $\mathrm{mm}$

CUADRO II. EVALUACIÓN DE LA APTITUD RECREATIVA DE LA PLAYA EL COCHÓRIT CON BASE EN LOS MÉTODOS DE PERCEPCIÓN DE USUARIOS E INDICADORES BIOFÍSICOS

\begin{tabular}{lcllc}
\hline Método & Infraestructura y servicios & Ambiente & Limpieza & Total \\
\hline Percepción de usuarios & 0.60 (medio) & 0.80 (alto) & 0.73 (medio) & 0.71 (medio) \\
Indicadores biofísicos & 0.51 (medio) & 0.53 (medio) & 0.37 (medio) & 0.47 (medio) \\
\hline
\end{tabular}

embargo, la percepción de los usuarios en su mayoría jóvenes, fue que la playa es sucia. Además, no hay recipientes para tirar la basura, lo que significa un incumplimiento de la normatividad (SEMARNAT 2016). Estudios previos han mostrado que los usuarios consideran que la limpieza o contaminación en las playas está determinada por factores visuales (Dinius 1981, Smith et al. 1991, Anfuso et al. 2014) y en la playa El Cochórit, un rasgo físico destacable como es la arena (Pranzini y Vitale 2011), fue valorada con mala limpieza. Lo anterior sugiere una falta de interés por el cuidado ambiental de la playa, así como un desconocimiento de riesgos sanitarios a la salud pública, tanto por usuarios como por prestadores de servicios y autoridades municipales de Empalme. Lo anterior debido a que nunca se ha implementado un programa de monitoreo de la calidad sanitaria de la playa. Estos resultados destacan un asunto de atención prioritario a considerar en su manejo, debido a que se ha observado que la limpieza de las playas es el principal atributo de satisfacción para los usuarios (Madanes et al. 2010, Botero et al. 2013).

El agua fue el atractivo más importante de visita y motivación para regresar a la playa, lo cual también ha sido observado como relevante en la percepción de usuarios en otras playas recreativas (Vaz et al. 2009), por lo que esta información es importante al considerar un proceso de certificación de playas (Yepes 1999).

Por otro lado, los usuarios visitan la playa durante todo el año, pero con mayor afluencia en semana santa y verano. Su percepción es que la playa está empeorando respecto a su última visita. Esto hace necesario diseñar un programa de mejoramiento, mantenimiento, monitoreo y fomento de la participación ciudadana con vigencia anual porque es un sitio visitado durante todo el año, aunque los flujos se incrementan en los periodos vacacionales arriba 
mencionados. La carencia de un programa de manejo en la playa El Cochórit, es característico en la mayoría de las playas recreativas de México, las cuales tienen presión antrópica de diferente índole durante todo el año y que frecuentemente deriva en efectos adversos ambientales y sanitarios (Espejel et al. 2007, Cervantes et al. 2008).

En la evaluación de playas recreativas (Roca et al. 2009) el componente de infraestructura y servicios asociados es importante. En opinión de los usuarios, la playa El Cochórit tiene limitaciones de estacionamiento y de accesos para personas discapacitadas, lo que muestra la necesidad de una infraestructura básica. Asimismo, se observó que los servicios públicos fueron inexistentes o insuficientes en calidad y cantidad de sanitarios y regaderas, lo que implica efectos potenciales adversos en la calidad sanitaria y riesgos en la salud pública (Colford et al. 2007, Molina-López et al. 2014).

El hecho de que la mayoría de los usuarios visiten la playa en familia, lo cual también fue observado en playas del Caribe por Botero et al. (2013), revela un servicio que estos lugares proveen a la sociedad y que resulta clave mantener.

Estos resultados aportaron evidencia de que la evaluación basada en la percepción de los usuarios proporciona elementos de juicio para el manejo de la playa.

\section{Aspectos biofísicos}

La playa de El Cochórit carece de infraestructura turística, equipamiento y servicios, lo que limita su aptitud recreativa. Los indicadores de infraestructura y servicios mostraron que no cumple los requisitos y especificaciones de sustentabilidad de calidad de playas establecidos en la norma mexicana NMXAA-120-SCFI-2016 (SEMARNAT 2016). El procedimiento para certificación sólo es aplicable en playas que cuentan con infraestructura y servicios tales como: sanitarios, basureros, señalización, etc. (Cervantes 2008).

Con base en la mayoría de los indicadores del componente ambiental, la playa El Cochórit no coincide con la "playa ideal" sugerida por Popoca y Espejel (2009). No obstante, es apta para actividad recreativa porque cuenta con características particulares y procesos costeros que determinan su uso. Por ejemplo, es somera con pendiente baja, casi nivelada con la superficie del piedemonte, el cual parece extenderse por debajo del nivel del mar, además está limitada por dunas activas de baja altura y planicies de inundación, no existe un proceso de erosión perceptible debido a la hidrodinámica del lugar, lo que aunado a su morfología permite mantener una playa ancha, extensa y con arena durante todo el año. Esto contrasta con lo observado por Cervantes y Espejel (2008) en la playa de Oceanside, California, en la que se alimenta de forma artificial con arena para contrarrestar la erosión y ésto implica un gasto anual de $\sim 16$ millones de dólares.

El tipo de oleaje es considerado como indicador por garantizar la seguridad de los usuarios en el área de baño, es una variable que impacta en la comodidad y seguridad de los bañistas, así como en la estabilidad de la playa y la línea de costa (Popoca y Espejel 2009). La playa El Cochórit se caracteriza por oleaje bajo, lo que la califica como una playa segura. Sin embargo, el servicio de salvavidas es requerido y se carece de ello.

En esta playa, se desarrollan bermas con elevación menor a un metro, razón por la cual las gravas y guijarros están ausentes. Sin embargo, la composición granulométrica de la arena no corresponde a una playa ideal, debido a que son arenas finas $(0.13$ - 0.18 mm de diámetro), y por ser volátiles y adherirse al cuerpo de los usuarios crea una sensación desagradable.

En la playa El Cochórit es necesario un mayor entendimiento de los mecanismos físicos que operan en su formación y equilibrio, así como de su dinámica y patrones estacionales para adecuar las actividades que se pueden llevar a cabo en el lugar.

El color de la arena de la playa es café claro debido a su origen predominantemente terrígeno y esta coloración no concuerda con la definición de "playa ideal". El color de la arena es uno de los indicadores importantes de atracción, debido a que una playa con arena blanca o dorada se percibe por los usuarios como una playa limpia y saludable (Williams et al. 1993, Pereira et al. 2003, Roig 2003).

León-López et al. (2014), observaron que en esta playa, la concentración de enterococos en el agua durante y hasta seis semanas después del periodo vacacional de semana santa 2014 fue $>200 \mathrm{NMP} / 100 \mathrm{~mL}$, es decir superior a los límites permisibles indicados en la norma oficial NMX-AA-120-SCFI (SEMARNAT 2016) y por lo tanto con riesgos a la salud. La presencia de estas bacterias fue atribuible a la afluencia excesiva de usuarios y a la insuficiencia de servicios sanitarios en la playa, considerando que se carece de aportes por ríos y que en este periodo estacional no hay lluvias. Estas observaciones fueron realizadas en un periodo corto del año, por lo que es necesario implementar un monitoreo anual de la calidad sanitaria del agua, debido a su relevancia en la salud pública (Molina-López et al. 2014, Quilliam et al. 2015). 
Mensah et al. (2014), mencionan que características tales como, la limpieza, la ausencia de ruido, la seguridad física, la calidad de infraestructura y los servicios asociados a las playas son elementos claves para su elección. Esto indica la necesidad de realizar esfuerzos de gestión para mejorar la aptitud recreativa de la playa El Cochórit. Sin embargo, existen usuarios cuyas preferencias no están asociadas a la ausencia de ruido o falta de infraestructura y servicios, su selección está basada principalmente en la facilidad de acceso (Rangel-Buitrago 2013).

Los resultados aportaron evidencia de que la aptitud recreativa de la playa El Cochórit con base tanto en la percepción de los usuarios como en los indicadores biofísicos, fue de nivel medio. La integración de estos dos métodos de evaluación fue consistente y proporciona elementos acerca de las condiciones que prevalecen en el sitio. Además aportan información guía para la toma de decisiones en la gestión ambiental de la playa.

\section{CONCLUSIONES}

El análisis aportó evidencia de que las características naturales de la playa son atractivas para los usuarios de la región y que la aptitud recreativa requiere ser mejorada con atención prioritaria en: (1) inversión en infraestructura y servicios, (2) mantenimiento de limpieza, (3) implementación de programas de educación ambiental y (4) monitoreo anual de la calidad sanitaria del agua y de la arena.

La estrategia de gestión ambiental con la voluntad e iniciativa de las autoridades del municipio de Empalme, debe considerar incluir a la playa El Cochórit en el Programa de Playas Limpias de la Comisión Nacional del Agua con el propósito de orientar esfuerzos hacia su certificación de playa sustentable.

\section{AGRADECIMIENTOS}

Gisela García Morales agradece al Consejo Nacional de Ciencia y Tecnología por la beca otorgada para realizar sus estudios de doctorado. Se agradece al personal técnico del Centro de Investigaciones Biológicas del Noroeste, S. C.: a David Urías Laborín, por el apoyo técnico en campo para el levantamiento de perfiles de playa y a Edgar Alcántara Razo, por la edición de las figuras. Este estudio se realizó con financiamiento de los proyectos: Ecología y manejo de ecosistemas costeros del Centro de Investigaciones
Biológicas del Noroeste, S. C., (CONACyT Ciencia Básica 251919 y CONACyT-Redes Temáticas 269540).

\section{REFERENCIAS}

Anfuso G., Williams A. T., Cabrera-Hernández J. A. y Pranzini E. (2014). Coastal scenic assessment and tourism management in western Cuba. Tourism Manage. $42,307-320$.

DOI: 10.1016/j.tourman.2013.12.001

Botero C., Anfuso G., Williams A. T., Zielisnky S., Da Silva C. P., Cervantes O., Silva L. y Cabrera J. A. (2013). Reasons for beach choice: European and Caribbean perspectives. J Coastal Res. 1 (65), 880-885. DOI: $10.2112 /$ SI65-149.1

Casanova-Matera L. (2002). Topografía plana. Taller de Publicaciones de Ingeniería, Universidad de Los Andes, Mérida, Venezuela, 123 pp.

Cervantes R. O. D., Espejel I., Arellano E. y Delhumeau S. (2008). User's perception as a tool to improve urban beach planning and management. Environ Manage. 42, 249-264.

DOI: $10.1007 / \mathrm{s} 00267-008-9104-8$

Cervantes R. O. D. y Espejel I. (2008). Design of an integrated evaluation index for recreational beaches. Ocean Coast Manage. 51 (5), 410-419.

DOI: 10.1016/j.ocecoaman.2008.01.007

CONAGUA. (2015). Discurso inaugural. Memorias. XI Encuentro de Playas Limpias. Comisión Nacional del Agua, 20 agosto 2015, Ciudad de México, México, pp. 1-2. CD-ROM

Cochran W. G. y Cox G. M. (1965). Diseños experimentales. 3ra. ed. Trillas, Ciudad de México, México, 661 pp.

Colford J., Wade T., Schiff K., Wright C., Griffith J., Sandhu S., Burns S., Sobsey M., Lovelance M. y Weisberg S. (2007). Water quality indicators and the risk of illness at beaches with nonpoint source of fecal contamination. Epidemiology 18 (1), 27-35.

DOI: 10.1097/01.ede.0000249425.32990.b9

Dinius S. H. (1981). Public perceptions in water quality evaluation. Water Resour. Bull. 17 (1), 116-121.

DOI: $10.1111 / \mathrm{j} .1752-1688.1981 . t b 02594 . \mathrm{x}$

Douglas M. W., Maddox R. A., Howard K. y Reyes S. (1993). The Mexican monsoon. J. Climate 6, 1665-1677 pp. DOI: 10.1175/1520-0442(1993)006<1665:TMM> 2.0. $\mathrm{CO} ; 2$

Duvat V. (2012). Public perception of beach quality: lessons learnt from a French case study. Memorias. I Congreso Internacional de Calidad Ambiental en Playas Turísticas. Santa Marta, Colombia. Del 28 al 30 marzo, pp. 14-20. CD-ROM 
Espejel I., Espinoza-Tenorio A., Cervantes O., Popoca I., Mejía A. y Delhumeau S. (2007). Proposal for an integrated risk index for the planning of recreational beaches: use at seven Mexican arid sites. J. Coastal Res. 50, 47-51.

Folk R. L. y Ward W. C. (1956). Brazos River bar: A study on the significance of grain size parameters. J. Petrol. 27 (1), 3-26.

García E. (2004). Modificaciones al sistema de clasificación climática de Köppen. 5ta. ed. Instituto de Geografía, Universidad Nacional Autónoma de México, Ciudad de México, México, 98 pp.

Harris L., Nel R., Holness S. y Schoeman D. (2015). Quantifying cumulative threats to sandy beach ecosystems: A tool to guide ecosystem-based management beyond coastal reserves. Ocean Coast Manage. 110, 12-24. DOI: 10.1016/j.ocecoaman.2015.03.003

Hernández E. G. (2003). Criterios para evaluar la aptitud recreativa de las playas en México: una propuesta metodológica. Gaceta Ecológica 68, 55-68.

INEGI (1993). Estudio hidrológico del estado de Sonora. Instituto Nacional de Estadística Geografía e Informática. Aguascalientes, Aguacalientes, México, 182 pp.

León-López C. E., Arreola-Lizárraga J. A., MendozaSalgado R. y García-Hernández J. (2014). Evaluación de la calidad sanitaria del agua en la playa El Cochórit, Sonora, México. Memorias XXXIV Congreso Interamericano de Ingeniería Sanitaria y Ambiental, Tema Salud y Medio Ambiente. Monterrey, Nuevo León, México. Del 2 al 6 noviembre, pp. 560-568. CD-ROM.

Lubinsky D., Victoria N., Cervantes O., Espinoza T. A., Delhumeaus S. y Espejel I. (2009). El valor de dos playas turísticas de Ensenada, Baja California según la percepción de los usuarios. Manejo Gestión y Certificación de Playas 2 (2), 45-56.

Lucrezi S., Saayman M. y Van der Merwe P. (2016). An assessment tool for sandy beaches: A case study for integrating beach description, human dimension, and economic factors to identify priority management issues. Ocean Coast Manage. 121, 1-22.

DOI: 10.1016/j.ocecoaman.2015.12.003

Madanes N., Faggi A. y Espejel I. (2010). Comparación de valoraciones de playas argentinas según la edad de los usuarios. Calidad de Vida y Salud 3 (1), 3-24.

Mendoza-Cantú M. E. (1997). Regionalización geomorfológica y de paisaje de la Zona Costera entre Guaymas y Agiabampo, Sonora, México. Tesis de Maestría. Instituto Tecnológico y de Estudios Superiores de Monterrey, Campus Guaymas, Sonora, 147 pp.

Mensah M., Fusu-Mensah B. Y. y Yirenya-Tawiah D. (2014). Assessing public perception of beach quality for ecotourism development: A case study in Kokrobite beach in the Greater Accra Region, Ghana. J. Environ. Earth Sci. 4 (11), 58-66.

Micallef A., Williams A. T., Radic M. y Ergin A. (2004). Application of a novel bathing area evaluation technique- a case study of Croatian Island beaches. World Leisure Journal 46 (4), 5-21. DOI: $10.1080 / 04419057.2004 .9674370$

Molina-López M., García-Ruiz E., Espigares-Rodríguez E., Espigares-García M., Fernández-Crehuet M. y Moreno-Roldán E. (2014). Valoración del riesgo de contaminación de aguas recreativas. Vigilancia sanitaria de las playas en España. Hig. Sanid. Ambient. 14 (2), 1191-1195.

Navarro R. C., Espejel I., Calderón de la Barca G. N., Cervantes O. y Leyva A. C. (2012). Incorporación de la percepción de los usuarios en la certificación de playas limpias. Costas 1, 140-146.

Nelson C., Morgan R., Williams A. y Wood J. (2000). Beach awards and management. Ocean Coast Manage. 43 (1), 87-98.

DOI: 10.1016/S0964-5691(99)00068-X

Pereira L. C., Jiménez J. A., Madeiros C. y Marinho Da Costa R. (2003). The influence of environmental status of Casa Caiada and Rio Doce beaches (Brazil) on beach users. Ocean Coast Manage. 46 (11-12), 1011-1030. DOI: 10.1016/j.ocecoaman.2004.01.002

Pinto K., Hachich E., Sato M., Di-Bari M., Coelho M., Matté M., Lamparelli C. y Razzolini M. (2012). Microbiological quality assessment of sand and water from three selected beaches of South Coast, Sao Paulo State, Brazil. Water Sci Technol. 66 (11), 2475-2482. DOI: $10.2166 /$ wst.2012.494

Popoca A. I. y Espejel I. (2009). Propuesta de una metodología para evaluar playas recreativas con destino turístico. Manejo, Gestión y Certificación de Playas, 119 -130 [en línea]. http://www.ileanaespejel. com/uploads/1/1/3/3/11330338/popoca_y_espejel.pdf 01/06/2015

Pranzini E. y Vitale G. (2011). Beach sand colour: The need for a standardized assessment procedure. J. Coastal Res. 61, 66-69. DOI: 10.2112/SI61-001.67

Quilliam R. S., Kinzelman J., Brunner J. y Oliver D. M. (2015). Resolving conflicts in public health protection and ecosystem service provision at designated bathing waters. Ocean Coast Manage. 161, 237-242. DOI: 10.1016/j.jenvman.2015.07.017

Rangel-Buitrago N., Correa I. D., Anfuso G., Ergin A. y Williams A. T. (2013). Assessing and managing scenery of the Caribbean Coast of Colombia. Tourism Manage. 35, 41-58. DOI: 10.1016/j.tourman.2012.05.008

Rippy M. A., Franks J. P. S., Feddersen F., Guza R. T. y Warrick J. A. (2013). Beach nourishment impacts on bacteriological water quality and phytoplankton bloom 
dynamics. Environ. Sci. Technol. 47 (12), 6146-6154. DOI: $10.1021 / \mathrm{es} 400572 \mathrm{k}$

Roca E., Riera C., Miriam V., Fragell R. y Junyent R. (2008). A combined assessment of beach occupancy and public perceptions of beach quality: A case study in the Costa Brava, Spain. Ocean Coast Manage. 51 (12), 839-846. DOI: 10.1016/j.ocecoaman.2008.08.005

Roca E., Villares M. y Ortego M. I. (2009). Assesing public perceptions on beach quality according to beach user's profile: A case study in the Costa Brava, Spain. Tourism Manage. 30 (4), 598-607.

DOI: $10.1016 /$ j.tourman.2008.10.015

Roig i Munar F. X. (2003). Análisis de la relación entre capacidad de carga física y capacidad de carga perceptual en playas naturales de la isla de Menorca. Investigaciones Geográficas 31, 107-118.

Roldán-Quintana J., Mora-Klepeis G., Calmus T., Valencia-Moreno M. y Lozano-Santacruz R. (2004). El graben de Empalme, Sonora, México: magmatismo y tectónica extensional asociados a la ruptura inicial del Golfo de California. Revista Mexicana de Ciencias Geológicas, 21 (3), 320-334.

Rosales-Grano P. (2008). Modelos de predicción de oleaje y su aplicación en la ingeniería de costas. Informe Técnico Instituto Tecnológico de Guaymas (DGECYTM-S04C-PO-01-02-2008), Guaymas, Sonora, México, 76 pp.

Royse Ch.F. (1970). An introduction to sediment analysis. Arizona State University Publications, Phoenix, EUA, 180 pp.

Sardá R., Valls J. F., Pint J., Ariza E., Lozoya J. P., Fraguell R. M., Martí C., Rucabado J., Ramis J., Jiménez J. A.
(2015). Towards a new Integrated Beach Management System: The Ecosystem-Based Management System for Beaches. Ocean Coast Manage. 118 (Part B), 167-177.

DOI: 10.1016/j.ocecoaman.2015.07.020

SEMARNAT (2016). Norma Mexicana NMX-AA120-SCFI-2016. Requisitos y especificaciones de sustentabilidad de calidad de playas. Diario Oficial de la Federación. 7 de diciembre de 2016.

Silva-Iñiguez L. y Fischer D. W. (2003). Quantification and classification of marine litter on the municipal beach of Ensenada, Baja California, Mexico. Mar Pollut Bull. 46 (1), 132-138. DOI: 10.1016/S0025-326X(02)00216-3

Smith D. G., Cragg A. M. y Croker G. F. (1991). Water clarity criteria for bathing waters based on user perception. J. Environ. Manage. 33 (3), 285-299.

DOI: $10.1016 / \mathrm{S} 0301-4797(91) 80030-9$

Vaz B., Williams A. T., Pereira C. y Phillips M. (2009). The importance of user's perception for beach management. J. Coastal Res. 56, 1164-1168.

Williams A. T., Leatherman S. P. y Simmons S. L. (1993). Beach aesthetic values: the south west peninsula, UK. En: Interdisciplinary discussions of coastal Research and coastal management issues and problems. $(\mathrm{H}$. Sterr, J. Hofstede y H.P. Plag, Eds.). Peter Lang International Academic Publishers, Frankfurt, Alemania, pp. $240-250$.

Williams A. y Micallef A. (2009). Beach management principles and practice. Earthscan, Londres, Reino Unido, $444 \mathrm{pp}$.

Yépes P. V. (1999). Las playas en la gestión sostenible del litoral. Cuadernos de Turismo 4, 89-110. 